\title{
Allergic Reaction Following Insertion of Vancomycin Loaded in Bone Cement
}

\author{
Goh KLa , Nazri MYa, Ong $C L^{b}$ \\ aDepartment of Orthopaedics, Traumatology \& Rehabilitation, Kulliyyah of Medicine, International Islamic \\ University Malaysia, Jalan Hospital Campus, 25100 Kuantan, Pahang, Malaysia \\ bDepartment of Dermatology, Hospital Tengku Ampuan Afzan, Kuantan, Pahang, Malaysia
}

\begin{abstract}
Vancomycin bead is an important ancillary treatment for osteomyelitis caused by methicillin-resistant Staphylococcus aureus (MRSA). However, red-man syndrome, which can be a life-threatening complication of vancomycin, may occur from the use of vancomycin beads albeit rarely. We report our first case of red-man syndrome caused by vancomycin bead's insertion for chronic osteomyelitis. Symptomatic treatment was not effective and removal of the vancomycin beads seems to be the best treatment for this condition.
\end{abstract}

KEYWORDS: Vancomycin beads, allergic syndrome, osteomyelitis, anaphylactoid reaction

\section{INTRODUCTION}

Antibiotic cement is a common adjunctive treatment for osteomyelitis. Gentamicin beads are commercially available for the treatment of osteomyelitis but not against methicillin-resistant Staphylococcus aureus (MRSA). Hence the use of vancomycin beads prepared by hand has been widely used to combat the emerging bone and joint infections caused by MRSA. Redman syndrome is the most common hypersensitivity reaction caused by vancomycin. It is frequently due to rapid infusion of vancomycin but has never been reported as a result of vancomycin when given as local antibiotic. We report a case where the patient developed this complication after insertion of vancomycin beads for the treatment of infected fractures.

Corresponding author:

Nazri Mohd Yusof

Department of Orthopaedics, Traumatology \&

Rehabilitation,

Kulliyyah of Medicine,

International Islamic University Malaysia (IIUM),

Jalan Hospital Campus,

25100 Kuantan, Pahang,

Malaysia.

$+609-5706131$

+609-5144451(Fax)

Email: nazrimy2000@yahoo.com

\section{CASE REPORT}

A 42-year-old army officer, sustained closed comminuted fracture distal third right tibia with intra-articular involvement following motor vehicle accident with no other injuries. Initial management includes cross-ankle external fixation and plating of the fibula which was performed on the day 9 of admission. Following the surgery, necrotic patch developed over the anteromedial aspect of the wound. The fibular wound was left open primarily due to swelling and was closed two weeks later. Upon discharge, the wound was clean with no evidence of infection. However, six weeks later, wound breakdown occurred over the necrotic patch with bone exposed. Wound debridement was performed followed by vacuum dressing. Initial wound culture was reported as Pseudomonas aeruginosa and intravenous gentamicin was initiated. However, the bone was found to be non-viable several days later, and bone drilling was performed. Repeat culture revealed MRSA as the causative micro-organism and intravenous vancomycin $500 \mathrm{mg}$ tds was administered. The vancomycin was given for duration of two weeks and stopped as serum vancomycin level was above the toxicity level (36mg/L; normal: $10-15 \mathrm{mg} / \mathrm{L})$. Patient also developed mild nephrotoxicity (serum creatinine: 261umol/ L) which resolved after withholding the vancomycin. 


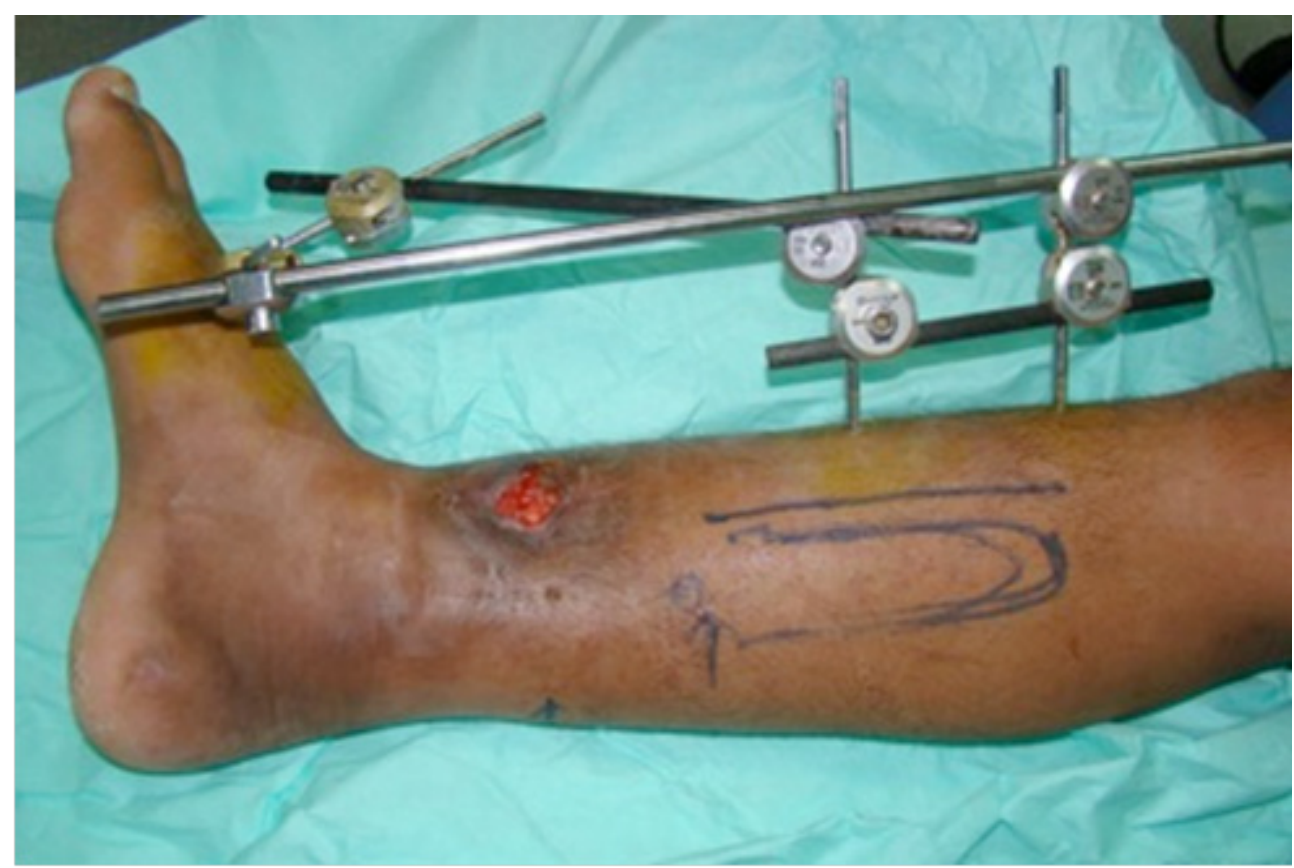

Figure 1. Wound over anteromedial aspect of the tibia prior to perforator flap surgery

As the non-viable bone progressed to osteomyelitis, bone excision, vancomycin beads insertion and perforator flap coverage was performed (Figure 1). The vancomycin bead was constituted by mixing $40 \mathrm{~g}$ bone cement (Smartset GHV Gentamycin bone cement, DePuy International Ltd, Blackpool, England) with 1 gram of powder vancomycin (Vancotex, Pharmatex Italia, Milano, Italy) and made into beads attached to a prolene suture. Following the surgery, patient began to develop pruritic rash all over the body as well as the operative site (Figure 2) but sparing the mucous membrane. As the rash was progressively worsening, dermatology opinion was sought and the diagnosis of red-man syndrome was made. Supportive therapy consisted of antihistamine, hydrocortisone cream and aqueous cream were prescribed, but the skin condition still persisted. However, his haemodynamic status was stable despite the severe skin problem. He did not improve until the vancomycin beads were removed ten days later. After removal of the vancomycin beads, the generalized rash reduced substantially and by the third day, he was discharged from the skin follow up. There was no evidence of any residual skin lesion and wound complications seen during the follow-up (Figure $3)$.

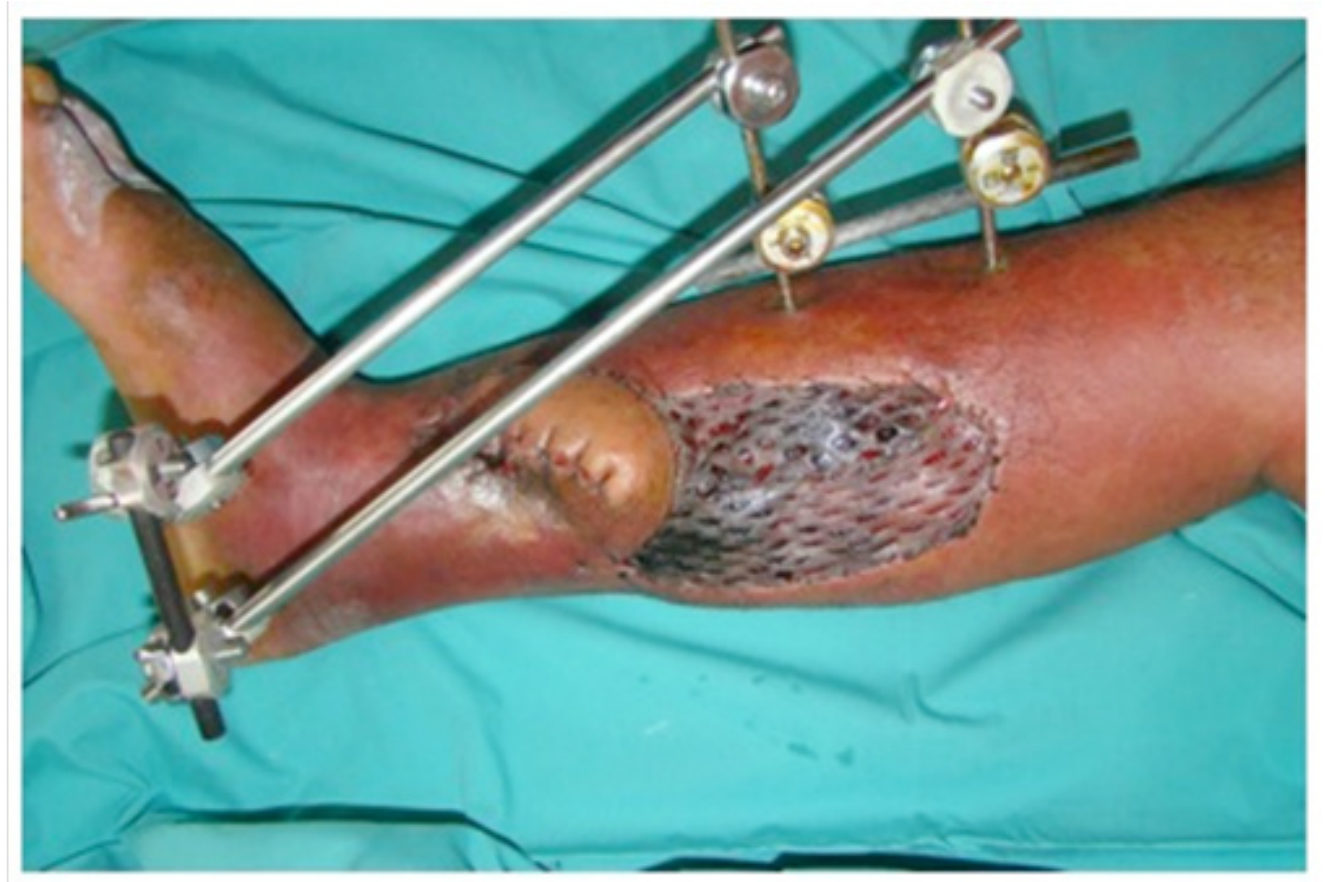

Figure 2. Erythematous rash which appeared on the leg after insertion of vancomycin beads 


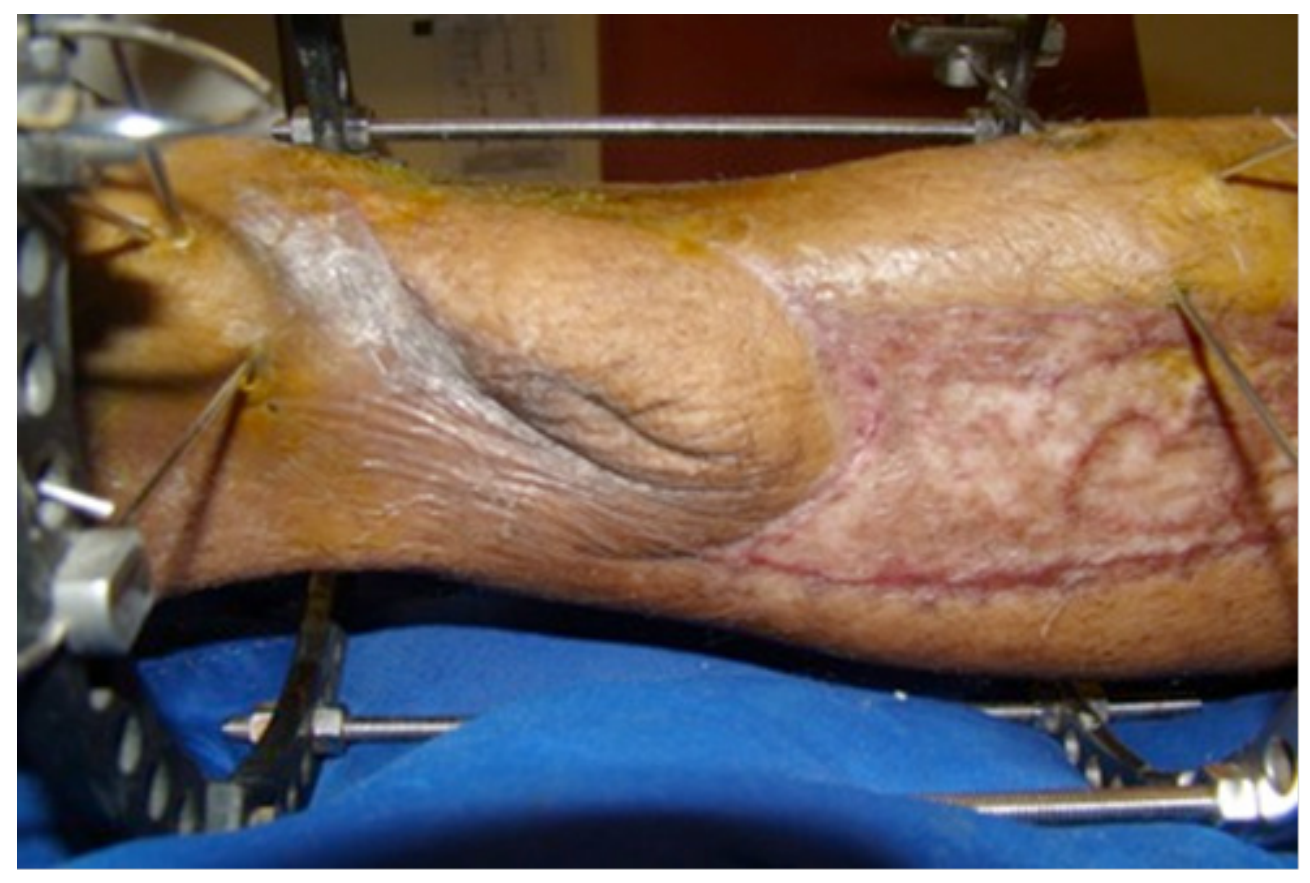

Figure 3. Erythematous rash disappeared after removal of vancomycin beads with good wound healing

\section{DISCUSSION}

Red man syndrome involves a pruritic, erythematous rash on the upper trunk, neck, and face associated with rapid vancomycin infusion, frequently in normal adults who receive $1000 \mathrm{mg}$ of vancomycin over 1 hour. ${ }^{1}$ This is a non-immunological or anaphylactoid reaction with the release of histamine as a result of degranulation of mast cells and basophils. The increase in plasma histamine concentration is attributed to the rate of vancomycin infusion and in turn, is correlated with the severity of the reaction. Other presentations of red-man syndrome include fever, dizziness, agitation, angioedema, tachycardia, hypotension, respiratory distress and cardiovascular collapse in severe cases.

The clinical presentation in this patient suggests redman syndrome, which usually results from a rapid infusion of vancomycin. However, he did not have this reaction when given intravenous vancomycin infusion earlier. The appearance of the reaction three days after the introduction of vancomycin beads, its persistence for eight days and its rapid resolution after removal of the beads all points out to the vancomycin beads as the likely cause. Allergic complication from insertion of vancomycin beads has never been reported before presumably because plasma absorption is minimal. This is confirmed by both animal and human study by Chohfi et al. ${ }^{2}$ who noted that although the local concentration was very high, the plasma level is 30 times lower than the toxic level.

The rate and mechanism of vancomycin delivery from bone cement is uncertain. Recent studies have shown that the porosity of the antibiotic cement plays a major role in defining the elution properties. Several factors that are thought to influence the elution properties include the type of cement used, the choice and amount of antibiotics added and the method of preparation. In vitro studies using continuous flow chamber to simulate in vivo condition have shown that the release kinetics of vancomycin, gentamicin and tobramicin from bone cement were similar. ${ }^{3}$ The elution of antibiotics from bone cement follows a biphasic pattern with an initial rapid phase followed by a slow secondary phase. The maximum release occurs within the first 24 hours and gradually stabilizes between 5 to 10 days. Even though experimental and clinical studies have shown a low systemic level in locally delivered vancomycin, ${ }^{4}$ vigilant monitoring for the first few days after vancomycin beads insertion for red-man syndrome is pertinent as in our case.

Vancomycin beads are usually prepared in conjunction with gentamicin cement powder due to its wide availability. In clinical practice, vancomycin in combination with gentamicin has been used to treat endocarditis due to its synergistic effect. The reported adverse reaction of this combination is renal impairment and there has not been any report of allergic reaction. ${ }^{5}$ Conversely; other possible causes of allergic reaction could arise from the cement components such as benzoyl peroxide, gentamicin and acrylates, which can be diagnosed by performing patch test. ${ }^{6,7}$

Vancomycin beads are an effective ancillary treatment for chronic infection with very few complications from allergy, toxicity or intolerance. ${ }^{8}$ The incidence of red man syndrome from vancomycin use is reported between $3.7 \%$ and $47 \%$ in patients with infection. ${ }^{9}$ However, the incidence with regards to vancomycin cement is unknown most likely due to its rare occurrence. 
The treatment of infusion-related red man syndrome consists of pre-medication and treatment with antihistamines such as chlorpheniramine or diphenhydramine. Several studies reported rapid dissolution with antihistamines and by witholding or reducing the rate of antibiotic delivery. ${ }^{10}$ In our experience, treatment with antihistamines and steroid cream were not effective as our patient did not show any improvement. Therefore, removal of the vancomycin beads seems to be the only effective method to treat this condition.

\section{REFERENCES}

1. McNamara DR, Steckelberg JM. Vancomycin. J Am Acad Orthop Surg 2005; 13:89-92.

2. Chohfi M, Langlais F, Fourastier J, et al. Pharma cokinetics, uses, and limitations of vancomycinloaded bone cement. Int Orthop 1998; 22:171-7.

3. Perry AC, Rouse MS, Khaliq Y, et al. Antimicrobial release kinetics from polymethylmethacrylate in a novel continuous flow chamber. Clin Orthop Relat Res 2002; 403:49-53.

4. Wahlig H, Dingeldein E, Bergmann R, Reuss K. The release of gentamicin from polymethylmethac rylate beads. An experimental and pharmacokinet ic study. J Bone Joint Surg Br 1978; 60:270-5.

5. Deresinski S. Vancomycin in combination with other antibiotics for the treatment of serious methicillin-resistant Staphylococcus aureus infec tions. Clin Infect Dis 2009; 49:1072-9.

6. Bircher A, Friederich NF, Seelig W, Scherer K. Allergic complications from orthopaedic joint implants: the role of delayed hypersensitivity to benzoyl peroxide in bone cement. Contact dermatitis 2012; 66:20-6.

7. Liippo J, Lammintausta K. Positive patch test reactions to gentamicin show sensitization to aminoglycosides from topical therapies, bone cements, and from systemic medication. Contact dermatitis 2008; 59:268-72.

8. Taggart T, Kerry RM, Norman P, Stockley I. The use of vancomycin-impregnated cement beads in the management of infection of prosthetic joints. J Bone Joint Surg Br 2002; 84:70-2.

9. Wazny LD, Daghigh B. Desensitization protocols for vancomycin hypersensitivity. Ann Pharmaco ther 2001; 35:1458-64.

10. Sahai J, Healy DP, Garris R, Berry A, Polk RE. Influ ence of antihistamine pretreatment on vanco mycin-induced red-man syndrome. J Infect Dis 1989; 160:876-81. 